\title{
COMPARISON OF DIFFERENT EXTRACTION METHODS FOR THE DETERMINATION OF PITURANTHOS SCOPARIUS ESSENTIAL OILS: CHEMICAL COMPOSITION, ANTIMICROBIAL AND ANTI- INFLAMMATORY ACTIVITIES
}

\section{Zahida Yasmina Hebbache ${ }^{\natural}$, Foudil-Cherif Yazid², Hichem Mohammedi², Ouahiba Belfadel², Fodili Mokhtar ${ }^{1}$}
${ }^{1}$ Laboratory of Organic Chemistry and Natural Substances, University Ziane Achour, City 05 July Road of Moudjbara, BP 3117, Djelfa, Algeria.
${ }^{2}$ Laboratory of Functional Organic Analysis, Department of Organic Chemistry, Faculty of Chemistry, University of Sciences and Technology Houari Boumediene (USTHB), El Alia, BP 32, Bab Ezzouar, Algeria.
هhebbachezahidayasmina@gmail.com

https://doi.org/10.34302/crpjfst/2021.13.3.13

Article history:

Received:

15 June 2021

Accepted:

12 August 2021

Keywords:

Pituranthos Scoparius;

Hydrodistillation, Microwave;

assisted extraction;

Steam distillation:

Anti-inflammatory activity;

Antimicrobial activity.

\section{ABSTRACT}

The essential oils of Pituranthos Scoparius obtained by hydrodistillation (HD), microwave assisted extraction (MAE) and steam distillation (SD) were investigated for their chemical components, oil yield diversity and microbial activity. As a result of this investigation, the anti-inflammatory activity of the essential oils is reported here for the first time. The essential oils extracted from P. Scoparius were analyzed by GC/MS. Sixty-three compounds were identified, representing $(87.69 \%)$ in HD, $(82.45 \%)$ in MAE, and $(88.94 \%)$ in SD of the essential oils' total compositions. The predominant compounds identified were Dillapiol (16.38-37.21\%), $\alpha$ Pinene (0.48-10.84\%) and Myristicin (4.21-9.37\%). The antimicrobial activity of the essential oils was Identified using the disk diffusion method against seven bacteria strains and two yeasts. An appreciable antimicrobial activity was observed against sarcina lutea, and weak activity was observed against staphylococcus epidermidis, saccharomyces cerevisiae and candida albicans. The anti-inflammatory activity of the essential oils was Identified using the carrageenan induced edema method. The essential oils extracted by HD, MEA and SD showed an anti-inflammatory activity comparable to Diclofenac. The results reveal that the method of extraction of P. scoparius influences the chemical composition and anti-inflammatory activity of the essential oils.

\section{Introduction}

The genus Pituranthos is a member of the Apiaceaes family and consists of more than 20 species (Ozenda, 2004). P. Scoparius (Coss \& Dur) Benth \& Hook is a plant endemic to the Saharan region of North Africa (Quezel and Santa, 1962) known as "Tattayet" in Tamahaq (Tuareg language) and "Guezzah" in Arabic.

$P$. Scoparius is a perennial aphyllous plant. The stems are $40-80 \mathrm{~cm}$ tall. The flowers, often with peduncle, white petals and marrow veins, are bunched in lateral umbels that are fairly spread out. However, due to the high temperature of the Central Sahara from which the plants used in this experiment were obtained, the species used did not contain leaves, flowers, or seeds (Quezel and Santa, 1962). P. Scoparius is used in traditional Algerian medicine for many purposes. In infusion, the aerial part is used as a remedy for digestive difficulties, diabetes, hepatitis, asthma and rheumatic diseases (Ould el hadj et al., 2003; (Hammiche 
and Maiza, 2006) and also as an additive flavoring (Boukef, 1986; Benchelah et al, 2000). The powdered stems are used against reptile bites (Benchelah et al., 2000; Abdellah and Sahki, 2004).

Previous research has shown that $P$. Scoparius is an important source of essential oils, with a considerable variation in major compounds depending on the part used, the plant maturity, the climate conditions and the extraction methods employed (Vernin et al., 1999; Vérité and Nacer, 2003; Smaili et al., 2011; Gourine et al., 2011; Lograda et al., 2013; Hammoudi et al., 2015; Belkacemi et al., 2015; Chikhoune et al., 2016; Ksouri et al., 2017; Malti et al., 2018, Attia et al., 2011). In addition, Sabinene and $\alpha$-pinene were identified as major compounds of most of $P$. Scoparius essential oils (Smaili et al, 2011; Lograda et al, 2013; Chikhoune et al., 2016; Malti et al., 2018; Attia et al, 2011). However, $\alpha$-pinene was not identified in samples from some Algerian Saharan regions (Ghardaia, Bechar and Tamenrasset) (Belkacemi et al., 2015; Ksouri et al., 2017; Malti et al., 2018) and Sabinene had not been reported in other studies on the same species (Vernin et al., 1999; Vérité and Nacer, 2003; Gourine et al., 2011; Hammoudi et al., 2015; Belkacemi et al., 2015; Ksouri et al., 2017). Alternatively, samples from Ghardaïa, Laghouat, Bechar, Tamenrasset and Batna were characterized by very high contents of limonene (Gourine et al., 2011; Ksouri et al., 2017; Malti et al., 2018). Bornyl Acetate was the dominant component of $P$. Scoparius essential oils from the Oum El Bouaghi and Tamenrasset regions (Vernin et al., 1999; Hammoudi et al., 2015). Other compounds were reported only in some samples and with appreciable amounts, such as: 7-methoxy-3-methyl-1-H-isichromen-1-one and methyl propene in P. Scoparius from Ghardaia (Belkacemi et al., 2015), 6- methoxyelemicine in samples from Ghardaïa, Bechar and Batna (Malti et al., 2018), Apiol in samples from Oum El Bouaghi (Vernin et al., 1999) and $\Delta$-3-Carene only in the Tunisian essential oil (Attia et al, 2011). Other essential oils were also characterized by a high presence of Myristicin
(Vérité and Nacer, 2003; Smaili et al., 2011; Gourine et al., 2011; Malti et al., 2018). These reported results confirmed the existence of different chemotypes in $P$. Scoparius essential oils.

Several studies reported the significant effect of the extraction methods on the yields and the chemical compositions of essential oils (Lucchesi et al., 2004: Elyemni et al., 2019; Lo Presti et al., 2005). Our work was based on the chemical variability of $P$. Scoparius essential oil as well as the antimicrobial and the antiinflammatory activities depending on the extraction methods. To the best of our knowledge, this is the first study Dedicated to extractions using the microwave assisted hydrodistillation is reported here for the first time. In addition, no pharmacological studies on the anti-inflammatory activity of essential oils were previously conducted. The aim of our work is to check the variation of the chemical composition, antimicrobial and antiinflammatory activities of the essential oils using three extraction methods (hydrodistillation, micro-wave and steam distillation).

\section{Materials and methods \\ 2.1. Samples}

The aerial parts of $P$. Scoparius were collected from the Algerian Sahara (Taessa, Tamanrasset) in July of 2012. The harvested plants were washed and then allowed to dry in the shade in a dry and ventilated place. Global Positioning System (GPS) technology was used to record the location: latitude $\left(23^{\circ} 05^{\prime} 64^{\prime \prime} \mathrm{N}\right)$, longitude $\left(5^{\circ} 30^{\prime} 79,7^{\prime \prime} \mathrm{E}\right)$ and altitude $(1748 \mathrm{~m})$. Identification of plant was performed in the Botany laboratory of the National Forest Research Institute in Tamanrasset (NFRI).

\subsection{Essential oils}

\subsubsection{Hydro-distillation (HD)}

$(200 \mathrm{~g})$ of plant were placed in a flask. The flask was then filled with (4L) of water. The flask containing the plant and water was brought to a boil for 4hours using Clevenger-type apparatus (Council of Europe, 2010). The 
essential oil-laden water vapors then passed through the refrigerant, condensed and fell into a separating funnel. The water and oil separated by density difference. All samples were collected and subsequently stored (sealed brown vial) at $4^{\circ} \mathrm{C}$ in the dark for further experimentation. The yield, based on the dry weight of the sample, was calculated.

\subsubsection{Microwave-assisted extraction (MAE)}

Microwave-assisted extraction was used at atmospheric pressure at $(850 \mathrm{~W}, 2450 \mathrm{MHz})$ power for 50 minutes (Beoletto et al., 2016), using a laboratory microwave for extracting the essential oil (M937, Samsung, United Kingdom). (100 g) of aerial part was placed in a (1L) flask with $(100 \mathrm{ml})$ of water and connected to the Clevenger-type apparatus located outside the microwave. The essential oil was collected in the same way as mentioned in the HD process. The yield, based on dry weight of the sample, was calculated.

\subsubsection{Steam distillation (SD)}

The steam was passed through a (5L) flask containing $(200 \mathrm{~g})$ of plant for $4 \mathrm{~h}$. The steam was then passed through a coiled tube where it was condensed. The distillate was collected. Finally, the essential oil obtained was separated through decantation using a separating funnel (Pushpangadan et al., 2012). The essential oil was collected in the same way as mentioned in the HD process. The yield, based on dry weight of the sample, was calculated.

\subsection{Gas chromatography (GC) and Gas chromatography-Mass spectrometry (GC- MS) analysis}

Analyses of the essential oils were carried out using a Hewlett-Packard 6800 GC equipped with an HP5-MS capillary column $(30 \mathrm{~m} \mathrm{x}$ $0.32 \mathrm{~mm} \times 0.25 \mathrm{~m}$ ) film thickness. The oven temperature was kept at $60^{\circ} \mathrm{C}$ for $8 \mathrm{~min}$ initially, then gradually increased to $250^{\circ} \mathrm{C}$ at $2{ }^{\circ} \mathrm{C} / \mathrm{min}$ and kept again at $250^{\circ} \mathrm{C}$ for $20 \mathrm{~min}$. The injector and detector temperature were $250{ }^{\circ} \mathrm{C}$ and 280 ${ }^{\circ} \mathrm{C}$, respectively. The carrier gas was helium used at flow rate of $1.2 \mathrm{~mL} / \mathrm{min}$. $1 \mu \mathrm{l}$ of sample was injected in split mode at a ratio of 1:70, where the detection ionization energy was 70
eV. For GC-MS analysis, the same abovementioned conditions for GC were applied. The mass scan range was 29-550 Uma. The percentage composition of the oils was expressed as peak area by integration from the total ion current. The percentage of each compound was computed using the normalization method from the GC peak area, calculated as mean value of three injections, without using correction factors. The identification of the compounds was based on the comparison of retention indices with those reported in the literature. Retention indices were calculated using the Van Den Dool and Kratz equation. The series of n-alkanes $\left(\mathrm{C}_{5}-\mathrm{C}_{28}\right)$ were injected in the same conditions. The EI-mass spectra of essential oils were compared with those of mass spectra library (NIST and Wiley $7 \mathrm{~N}$ library) and the literature (Adams, 2007).

\subsection{Pharmacological activities}

\subsubsection{Animals}

Strains of albino mice of both sexes, weighing (20-25g) were used for pharmacological studies. The animals were obtained from the Department of Pharmacotoxicology, SAIDAL AntibioticalMedea, Algeria. The animals were housed in cages under standard laboratory conditions (12hour light/dark cycle at $25^{\circ} \mathrm{C}$ ). The animals were divided into groups of five and fasted for 12 hours before the experiment.

\subsubsection{Acute toxicity assay}

The acute toxicity test was performed using the Lorke method (Lorke, 1983). Mice were divided into several groups of five. $(0.5 \mathrm{~mL})$ of (2000 mg / kg) of essential oils were administered orally. The control group was treated in the same manner without administering essential oil. The mice were observed for 14 days. The mice that died in each group were counted for lethal dose (LD50) determination.

\subsubsection{Anti-inflammatory activity}

The anti-inflammatory activity was determined based on the carrageenan induced edema test (Levy, 1969). Groups of five mice each were treated orally with $(0.5 \mathrm{~mL})$ of 
essential oils $(50 \mathrm{mg} / \mathrm{kg}, 100 \mathrm{mg} / \mathrm{kg}$ and 150 $\mathrm{mg} / \mathrm{kg})$. After 30 minutes, $(0.025 \mathrm{~mL})$ of $(1 \%)$ carrageenan solution (diluted with $0.9 \%$ physiological water) was injected into the right hind paw planter surface of the mice. Positive control received $(50 \mathrm{mg} / \mathrm{kg}, 100 \mathrm{mg} / \mathrm{kg}$ and 150 $\mathrm{mg} / \mathrm{kg}$ ) of Diclofenac. After 3.30 hours, the animal suffers euthanasia and their right and left paws were removed and weighed with a precision scale. The percentage of edema was calculated according to the following equation:

$\%$ Edema $=100 \times($ Average weight of left paws - average weight of right paws) $\div$ Average weight of left paws.

The percentage reduction of edema in the treated mice relative to the control was calculated according to the following equation:

$\%$ Reduction of edema $=100 \times(\%$ Edema control - \% Edema test $\div \%$ Edema control.

\subsubsection{Antimicrobial activity}

\subsubsection{Microbial strains}

Nine stains were used, including five Grampositive bacteria: Staphylococcus Aureus ATCC 6538/P, Staphylococcus Epidermidis ATCC 12228, Enterobacter Faecalis, Bacillus Subtilis ATCC 6633 and Sarcina Lutea, two Gramnegative bacteria: Escherichia Coli ATCC 11105 and Pseudomonas Aeruginosa ATCC 27853 and two yeasts: Candida Albicans ATCC 10231 and Saccharomyces Cerevisiae ATCC 2601.

The microbial strains belonged to the American Type Culture Collection, except for Enterobacter Faecalis, and were supplied from Frantz Fanon Hospital in Blida, Algeria and Pastor Institution in Algiers, Algeria.

\subsubsection{Agar disk diffusion method}

The antimicrobial activity was determined using the disk diffusion method (National Committee for Clinical Laboratory Standards, 1997).The test was performed using the Soja Agar medium for bacterial germs and Sabouraud medium for fungal germs. The microbial suspensions were adjusted to $10^{6} \mathrm{CFU} / \mathrm{ml}$
(Colony Forming Units) in a sterile saline solution and were streaked over the surface of the plates using a sterile cotton swab in order to get a uniform microbial growth on the test plates. Sterile filter paper disks $(6 \mathrm{~mm}$ in diameter) were then permeated with $(10 \mathrm{uL})$ of essential oil. Plates were incubated at $37^{\circ} \mathrm{C}$ for 24h. Penicillin G, Oxalin and Amoxypen (200 $\mathrm{mg} / \mathrm{mL}$ ) were used as standard antibiotics. The antimicrobial activity was evaluated by measuring the diameter of inhibition in $\mathrm{mm}$ (diameter of the disc included). Assays were performed in triplicate.

\subsection{Statistical analysis}

Collected data are expressed as mean \pm standard deviation. All tests were performed using IBM SPSS $v 23$. The comparison between chemical compositions was performed using a multivariate analysis of variance, sustained by a Tukey post hoc test, if significant. The study of biological activity was established by the ANOVA or Kruskal Wallis tests, sustained by Tukey or Mann Whitney U tests, if significant. Differences are considered as significant when $(p<0.05)$, highly significant when $(p<0.01)$ or strongly significant when $(p<0.001)$.

\section{Results and discussions}

\subsection{Yields and chemical composition of essential oils}

The extraction of the aerial parts of $P$. Scoparius produced yellow essential oils ranging in yield from $0.20 \%$ to $0.53 \%(\mathrm{w} / \mathrm{w})$ as a function of the extraction method (Table 1). The results showed that the extraction method had a significant influence on essential oil yields of $P$. Scoparius $(\mathrm{p}<0.05)$. The extraction by HD provided the highest yield $(0.53 \%, 4 \mathrm{~h})$ compared to that obtained by SD $(0.33 \%, 4 \mathrm{~h})$, however the lowest yield was obtained using MAE $(0.20 \%, 0.5 \mathrm{~h})$. The results obtained agreed with those reported by several authors (0.25-0.99\%) (Vérité and Nacer, 2003; Lograda et al., 2013; Hammoudi et al., 2015; Ksouri et al., 2017; Malti et al, 2018, Attia et al., 2011). However higher oil yields were obtained from some different Algerian Saharan regions in 
others studies (1.0-2.8\%) (Gourine et al., 2011;

Lograda et al., 2013).

Table 1. Variability in the chemical composition of $P$. Scoparius essential oil obtained by different methods

\begin{tabular}{|c|c|c|c|c|c|c|}
\hline No. & Compounds $^{a}$ & $\mathbf{R I}^{\mathbf{b}}$ & $\mathbf{R I}^{\mathbf{c}}$ & HD & MAE & SD \\
\hline 1 & $\alpha$-Thujene & 914 & 930 & 1.94 & - & 0.49 \\
\hline 2 & $\alpha$-Pinene & 925 & 939 & 10.84 & 0.48 & 3.28 \\
\hline 3 & Camphene & 937 & 953 & 0.16 & - & $\operatorname{tr}$ \\
\hline 4 & Sabinene & 967 & 976 & 0,88 & $\operatorname{tr}$ & 0.26 \\
\hline 5 & $\beta$-Pinene & 971 & 979 & 2.63 & 0.27 & 1.19 \\
\hline 6 & $\beta$-Myrcene & 989 & 990 & 0.38 & - & 0.14 \\
\hline 7 & I-Phellandrene & 1003 & 1005 & 0.39 & $\operatorname{tr}$ & 0.18 \\
\hline 8 & $\Delta$-3-Carene & 1009 & 1011 & 1.08 & $\operatorname{tr}$ & 0.80 \\
\hline 9 & $\alpha$-Terpinene & 1015 & 1017 & 0.28 & $\operatorname{tr}$ & 0.25 \\
\hline 10 & $p$-Cymene & 1025 & 1024 & 4.89 & 1.57 & 4.02 \\
\hline 11 & Limonene & 1028 & 1029 & 3.16 & 0.77 & 1.73 \\
\hline 12 & $\beta$-Ocimene $Z$ & 1039 & 1040 & 2.70 & 0.32 & 1.00 \\
\hline 13 & $\beta$-Ocimene $E$ & 1047 & 1050 & 0.27 & - & $\operatorname{tr}$ \\
\hline 14 & $\gamma$-Terpinene & 1057 & 1059 & 0.56 & 0.18 & 0.60 \\
\hline 15 & Cissabinene hydrate & 1064 & 1068 & 0.11 & $\operatorname{tr}$ & $\operatorname{tr}$ \\
\hline 16 & $\alpha$-Terpinolene & 1085 & 1088 & 0.24 & 0.19 & 0.38 \\
\hline 17 & $\begin{array}{l}\text { Transsabinene } \\
\text { hydrate }\end{array}$ & 1095 & 1097 & 0.22 & - & 0.13 \\
\hline 18 & $\beta$-Thujone & 1113 & 1114 & 0.16 & - & 0.30 \\
\hline 19 & $\alpha$-Campholenal & 1117 & 1125 & - & 0.65 & - \\
\hline 20 & Terpinene-1-ol & 1118 & - & 0.15 & - & - \\
\hline 21 & $\alpha$-Comphoaldehyde & 1123 & - & 0.37 & - & 0.53 \\
\hline 22 & Pinocarveol (trans) & 1135 & 1139 & 0.69 & 0.92 & 0.57 \\
\hline 23 & Cis Verbenol & 1138 & 1140 & 0.11 & 0.49 & - \\
\hline 24 & Trans Verbenol & 1142 & 1144 & - & - & 0.27 \\
\hline 25 & Penthylbenzene & 1154 & 1156 & 0.14 & - & 0.27 \\
\hline 26 & Pinocarvone & 1160 & 1162 & 0.55 & 0.52 & 0.30 \\
\hline 27 & 4-Terpineol & 1176 & 1177 & 1.04 & 1.10 & 1.36 \\
\hline 28 & Crypton & 1179 & - & - & 0.22 & 0.26 \\
\hline 29 & Cymen-8-ol-para & 1183 & 1183 & 0.30 & 0.37 & 0.44 \\
\hline 30 & $\alpha$-Terpineol & 1189 & 1189 & 0.18 & - & 0.29 \\
\hline 31 & (-)Myrtenal & 1194 & 1193 & 0.44 & 0.37 & 0.24 \\
\hline 32 & Myrtenol & 1200 & 1200 & 0.48 & 0.36 & 0.25 \\
\hline 33 & $\begin{array}{l}\alpha \text {-Phellandrene } \\
\text { epoxide }\end{array}$ & 1204 & - & - & 0.67 & 0.70 \\
\hline 34 & $I$-Verbenone & 1206 & 1205 & 0.21 & 0.32 & 0.34 \\
\hline 35 & TransCarveol & 1218 & 1216 & 0.30 & 0.56 & 0.41 \\
\hline 36 & $\begin{array}{l}\text { Propanal,2-methyl-3- } \\
\text { phenyl }\end{array}$ & 1238 & - & - & - & 0.41 \\
\hline 37 & I-Carvone & 1241 & 1242 & 0.13 & 0.24 & 0.15 \\
\hline
\end{tabular}


Hebbache et al./ Carpathian Journal of Food Science and Technology, 2021, 13(3), 158-172

\begin{tabular}{|c|c|c|c|c|c|c|}
\hline 38 & Carvotanacetone & 1245 & 1247 & $\operatorname{tr}$ & 0.14 & 0.12 \\
\hline 39 & Phellandral & 1273 & 1271 & 0.10 & - & 0.20 \\
\hline 40 & Safranal & 1280 & - & - & 0.68 & - \\
\hline 41 & $p$-Cymen-7-ol & 1287 & 1290 & $\operatorname{tr}$ & 0.18 & 0.22 \\
\hline 42 & Thymol & 1289 & 1290 & - & - & 0.15 \\
\hline 43 & Carvacrol & 1300 & 1299 & 0.30 & 0.61 & 0.73 \\
\hline 44 & $\alpha$-Copaene & 1370 & 1376 & 0.11 & 0.18 & 0.22 \\
\hline 45 & Methyleugenol & 1400 & 1401 & 0.25 & 0.61 & 0.37 \\
\hline 46 & $\beta$-Selinene & 1481 & 1485 & 0.21 & 0.11 & 0.22 \\
\hline 47 & Myristicin & 1523 & 1520 & 4.21 & 9.35 & 7.15 \\
\hline 48 & $\alpha$-Calacorene & 1533 & 1545 & $\operatorname{tr}$ & 0.11 & 0.18 \\
\hline 49 & $\beta$-Calacorene & 1549 & - & 0.39 & - & - \\
\hline 50 & Élémicine & 1556 & 1554 & 0.14 & 0.33 & 0.28 \\
\hline 51 & $\begin{array}{l}\text { 1,5-epoxysalvial-4 } \\
\text { (14) ene }\end{array}$ & 1560 & - & 0.62 & 0.60 & 0.66 \\
\hline 52 & Spathulenol & 1575 & 1576 & 4.27 & 5.23 & 4.23 \\
\hline 53 & $\begin{array}{l}\text { Salvia-4 (14)-en-1- } \\
\text { one }\end{array}$ & 1586 & - & 0.44 & 0.76 & 0.71 \\
\hline 54 & Caryophyllene oxide & 1600 & 1581 & 0.57 & - & 0.24 \\
\hline 55 & Dillapiol & 1612 & 1622 & 16.38 & 37.21 & 31.95 \\
\hline 56 & Isospathulenol & 1634 & - & 0.52 & 0.18 & 0.22 \\
\hline 57 & $\beta$-eudesmol & 1645 & 1649 & 9.19 & 7.10 & 7.70 \\
\hline 58 & ButylidenephtalideZ & 1668 & 1672 & 7.15 & 1.23 & 6.65 \\
\hline 59 & Butylidenephtalide $E$ & 1700 & 1718 & 1.86 & 2.72 & 2.20 \\
\hline 60 & 3-N-Butyl phtalide & 1724 & 1739 & 4.46 & 3.50 & 2.86 \\
\hline 61 & $\begin{array}{l}\text { Butylidenedihydro- } \\
\text { phtalide }\end{array}$ & 1777 & - & 0.33 & - & 0.14 \\
\hline 62 & $\begin{array}{l}\text { Hexahydrofarnesyl } \\
\text { acetone }\end{array}$ & 1848 & - & - & 0.32 & - \\
\hline 63 & Acidepalmitique & 1947 & - & 0.21 & 0.73 & $\operatorname{tr}$ \\
\hline \multicolumn{2}{|c|}{ Yield } & & & 0.53 & 0.20 & 0.33 \\
\hline \multicolumn{2}{|c|}{ Monoterpene Hydrocarbons } & & & 30.16 & 3.59 & 13.94 \\
\hline \multicolumn{2}{|c|}{ Oxygenated Monoterpenes } & & & 6.22 & 8.59 & 9.02 \\
\hline \multicolumn{2}{|c|}{ Sesquiterpene Hydrocarbons } & & & 5.17 & 10.36 & 8.14 \\
\hline \multicolumn{2}{|c|}{ Oxygenated Sesquiterpenes } & & & 32.13 & 51.41 & 45.99 \\
\hline \multicolumn{2}{|c|}{ Phtalides } & & & 13.8 & 7.45 & 11.85 \\
\hline \multicolumn{2}{|c|}{ Others } & & & 0.21 & 1.05 & $\operatorname{tr}$ \\
\hline \multicolumn{2}{|c|}{ Total } & & & 87.69 & 82.45 & 88.94 \\
\hline
\end{tabular}

${ }^{\mathrm{a}}$ Compounds listed according to their elution order on apolar HP5MS ${ }^{\mathrm{TM}}$ capillary, ${ }^{\mathrm{b}}$ Retention Indices on apolar column (HP5MS), ${ }^{\mathrm{c}}$ Retention indices of literature on apolar column reported by Adams (2007), tr: trace $(<0.1 \%)$,

-: Not detected, all components were identified by comparison of their retention indices with those of published data (Adams, 2007) and mass spectra with literature data, the MS library (NIST and Wiley 7N library)

GC and GC-MS analyses resulted in the identification of sixty-three compounds representing (82.45-88.94\%) of the total composition of the essential oils. As shown in
(Table 1), a noteworthy qualitative similarity was observed, but with differences in the abundance of major compounds. The oxygenated sesquiterpenes were found to be the 
main chemical group in all samples (32.13$51.41 \%)$. The second most frequently identified chemical class in the hydrodistillated sample was monoterpene hydrocarbons (30.16\%). This fraction was present in much lower amounts in the samples obtained by SD and MAE (13.94 and $3.59 \%$, respectively). The oxygenated monoterpene compounds were present in lower amounts in all samples $(<10 \%)$. Among the three extractive processes, an important observation revealed that MAE isolated the highest relative amount of oxygenated sesquiterpenes $(51.41 \%)$, as compared to SD with $(45.99 \%)$ and HD with $(32.13 \%)$. This result, which indicates that oils isolated by Microwave-assisted hydrodistillation are characterized by a higher content of oxygenated compounds, was previously reported on essential oils of three different species (Ocimum basilicum L., Mentha crispa L. and Thymus vulgaris L.) (Lucchesi et al., 2004). The same finding was obtained on Rosmarinus officinalis L. essential oils by (Elyemni et al., 2019; Karakayat et al., 2014; Okoh et al., 2010; Moradi et al., 2018). This may be due to an increase in hydrothermal effects in HD, compared to MAE which uses a lower quantity of water, that is rapidly heated [20, 30].Conversely, Lo Presti, M.et al. reported in their study on the same species (Rosmarinus officinalis L.) that the essential oils produced by MAE and HD were characterized by very similar chemical profiles (Lo Presti et al., 2005). Alternatively, a different result was previously published, where the amount of the oxygenated compounds was higher in the hydrodistillated essential oil compared with the oil isolated by microwave distillation from dried Cuminum cyminum L., and Zanthoxylum bungeanum Maxim (Wang et al., 2006). This contradiction in results is probably due to the fact that the amount of oxygenated compounds in essential oil is not dependent only on extraction methods, but also on many other factors including: species used, plant maturity, plant part used and harvest site (Mohammedi et al., 2015; Mohammedi et al., 2019).
The major compounds identified in the three essential oils were Dillapiol, $\beta$-eudesmol, Myristicin, $\alpha$-Pinene and Butylidene phtalide Z, but with significant differences in their proportions depending on the extraction method used. Dillapiol, the major compound in all samples, was present at (37.21\%) and (31.95\%), respectively, for MAE and $\mathrm{SD}$, but only at $(16.38 \%)$ for HD. In the same way, Myristicin, the second compound in MAE oil (9.35\%), amounted to $(7.15 \%)$ and $(4.21 \%)$ in the SD and HD samples, respectively. The hydrodistillated oil contained the highest amount of $\alpha$-Pinene $(10.84 \%)$, which is present in much lower amount in the other oils obtained by SD and MAE (3.28\% and $0.48 \%$, respectively). Both samples extracted by HD and SD contained important amounts of Butylidene phtalide $\mathrm{Z}$ (7.15\% and $6.65 \%$, respectively) and p-cymene $(4.89 \%$ and $4.02 \%$, respectively). These compounds account for only (1.23-1.57\%) in the oil obtained by MAE. Other major compounds were determined with equivalent amounts in the oils extracted by HD, SD and MAE such as $\beta$ eudesmol $(9.19 \%, \quad 7.70 \%$ and $7.10 \%$, respectively), Spathulenol $(4.27 \%, 4.23 \%$ and $5.23 \%$, respectively) and $3-\mathrm{N}-$ Butyl phtalide (4.46\%, 2.86\% and $3.50 \%$, respectively). To the best of our knowledge, 3-N-Butyl phtalide has never been identified in P. Scoparius essential oils.

The multivariate analysis of variance showed a strong significant effect of methods on major essential oil compounds. $(p<0.001)$.Extraction method variation indicates a significant effect on: Dillapiol $(p<0.001) ; \alpha-$ Pinene $(p<0.001) ; \quad$ Butylidene phtalide $Z$ $(p<0.001) ; \beta$-Ocimene $Z(p<0.001) ; \beta$-Pinene $(p<0.001) ; \quad \alpha$-Thujene $(p<0.001) ; \quad$ Myristicin $(p<0.001) ; \quad$ Limonene $(p<0.001) ; \quad p$-Cymene $(p<0.001) ; 3-N-B u t y l$ phtalide $(p<0.001) ; \beta-$ eudesmol $(p<0.001)$; Spathulenol $(p<0.001) ; \Delta$ 3 -carene $(p<0.001) ;$ Butylidene phtalide $E$ $(p<0.001)$; 4-terpineol $(p=0.002)$. The mean comparison between major compounds was assessed to choose the best extraction method for each Substance. Results show a considerable difference between methods for most 
compounds, except for 4-terpineol, for which results indicate no difference in SD vs MAE $(\mathrm{p}=0.074)$, as well as Spathulenol, for which results indicate no difference in SD vs HD $(\mathrm{p}=0.887)$.

Table 2. Major compounds of $P$. Scoparius essential oils from different origins previously reported.

\begin{tabular}{|c|c|c|c|c|c|c|c|c|c|}
\hline \multirow{2}{*}{\multicolumn{2}{|c|}{$\begin{array}{l}\text { Country / } \\
\text { Region }\end{array}$}} & \multirow[b]{2}{*}{ 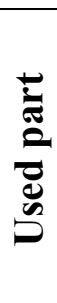 } & \multicolumn{6}{|c|}{ Main constituents (\%) } & \multirow[b]{2}{*}{$\cong$} \\
\hline & & & 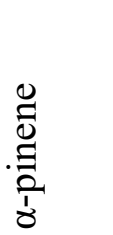 & 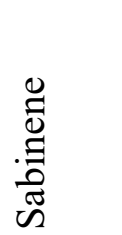 & 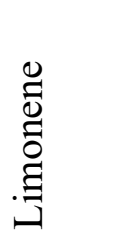 & 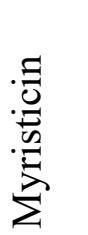 & 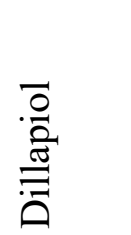 & 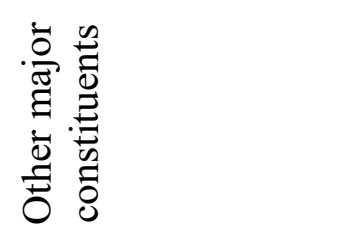 & \\
\hline & Oum El & $\mathrm{S}$ & 34 & - & - & - & - & Apiol (15.0) & \multirow[b]{2}{*}{ 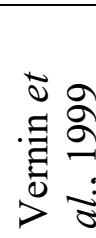 } \\
\hline & Bouaghi & $\mathrm{d}$ & 11 & - & - & - & - & $\begin{array}{l}\text { Apiol (52.8); } \\
\text { Bornyl acetate } \\
(21 \%) .\end{array}$ & \\
\hline \multirow{2}{*}{\multicolumn{2}{|c|}{ Ghardaïa }} & $\mathrm{S}$ & 6.8 & - & 9.8 & 7.2 & - & $\begin{array}{l}\text { Germacrene D } \\
\quad(12.7) ; \\
\alpha \text {-Phellandrene } \\
\text { (7.1); Methyl } \\
\text { eugenol (5.9). }\end{array}$ & \multirow{2}{*}{ 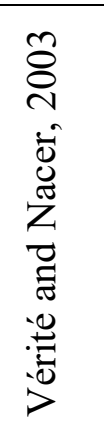 } \\
\hline & & $\mathrm{d}$ & 8.2 & - & 11.2 & 11.1 & - & $\begin{array}{l}\text { Caryophyllene } \\
\text { oxide (12.2); } p \text { - } \\
\text { Cymene (7.5); } \\
\text { Thymol (5.9). }\end{array}$ & \\
\hline \multirow{7}{*}{ 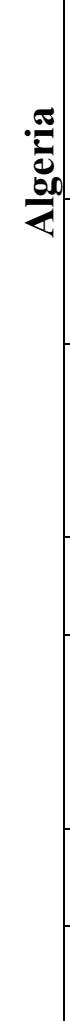 } & M'sila & $\mathrm{F}$ & 17.4 & 7.5 & - & 24.1 & - & $\begin{array}{c}\alpha \text {-Phellandrene } \\
\text { (15.6). }\end{array}$ & 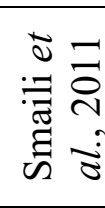 \\
\hline & Ghardaïa & $\mathrm{P}$ & $\begin{array}{l}4.4- \\
11.2\end{array}$ & - & $\begin{array}{l}32.7- \\
66.5\end{array}$ & $\begin{array}{c}\leq \\
31.1\end{array}$ & $\leq 23.0$ & $\begin{array}{c}\alpha \text {-Phellandrene }(\leq \\
6.4) ; \text { Germacrene D } \\
(\leq 6.3) .\end{array}$ & \multirow{3}{*}{ 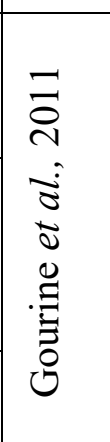 } \\
\hline & Djelfa & $\mathrm{P}$ & $\begin{array}{l}23.7- \\
27.0\end{array}$ & - & $1.0-7.8$ & $\begin{array}{c}\leq \\
18.2\end{array}$ & $\leq 47.3$ & $\begin{array}{c}\text { Bornyl acetate }(\leq \\
9.6) ; \\
p \text {-Cymene }(2-6.7) \\
\beta \text {-Pinene }(\leq 5.3)\end{array}$ & \\
\hline & Laghouat & $\mathrm{P}$ & $\begin{array}{l}35.1- \\
35.8\end{array}$ & - & $\begin{array}{l}7.0- \\
30.0\end{array}$ & - & $\begin{array}{l}9.9- \\
25.7\end{array}$ & $\begin{array}{l}\text { Bornyl acetate (3.0- } \\
9.5) ; \beta \text {-Pinene (5.2). }\end{array}$ & \\
\hline & M'sila & $\mathrm{P}$ & 16.4 & 14.8 & - & - & - & $\begin{array}{c}\text { Caryophyllene } \\
\text { oxide (9.7); } \alpha- \\
\text { Farnesene (7.7); } \\
\alpha \text {-Terpinene (5.8). }\end{array}$ & $\begin{array}{l}\frac{m}{\tilde{N}} \\
\stackrel{z}{\tilde{z}}\end{array}$ \\
\hline & Batna & $\mathrm{P}$ & 23.3 & 18.6 & - & - & - & $\begin{array}{c}\alpha \text {-Terpinene (7.7); } \\
\beta \text {-Ocimene-E (7.6). }\end{array}$ & $\begin{array}{l}\widetilde{0} \\
\frac{\pi}{\pi}\end{array}$ \\
\hline & Biskra & $P$ & $\begin{array}{l}8.3- \\
13.4\end{array}$ & $\begin{array}{l}18.9- \\
24.8\end{array}$ & - & 7.6 & $\begin{array}{l}6.6- \\
16.8\end{array}$ & - & 兽 \\
\hline
\end{tabular}




\begin{tabular}{|c|c|c|c|c|c|c|c|c|}
\hline $\begin{array}{l}\text { Tamenra } \\
\text {-set }\end{array}$ & $\mathrm{P}$ & 12.1 & - & - & - & - & $\begin{array}{c}\text { Bornyl acetate (32); } \\
\text { Epi- } \\
\text { Bicycle } \\
\text { sesquiphellandrene } \\
\text { (8.4); Eremophilene } \\
\text { (8.2); } \\
\text { y-Cadinène (6.3). }\end{array}$ & 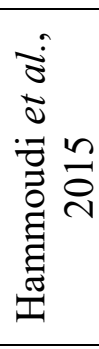 \\
\hline Ghardaïa & $\mathrm{P}$ & - & - & - & 12.1 & - & $\begin{array}{l}\text { 7-Methoxy-3- } \\
\text { methyl-1-H- } \\
\text { isichromen-1-one } \\
\text { (10.6); Methyl } \\
\text { propene (9.9). }\end{array}$ & 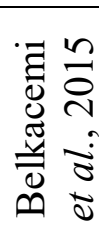 \\
\hline $\begin{array}{l}\text { Souk } \\
\text { Ahras }\end{array}$ & $\mathrm{P}$ & $\begin{array}{l}16.3- \\
26.5\end{array}$ & $\begin{array}{l}23.6- \\
34.4\end{array}$ & - & - & - & $\begin{array}{c}p \text {-Cymene }(8.6- \\
\text { 10.1); } \\
\text { Terpinen-4-ol }(4.1 \text { - } \\
\text { 9.7); } \alpha \text {-Thujene } \\
(5.3-5.7) ; \\
\beta \text {-Pinene }(\leq 5.5)\end{array}$ & 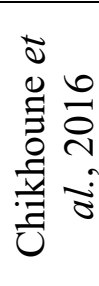 \\
\hline $\begin{array}{l}\text { Tamenra } \\
\text { s -set }\end{array}$ & $\mathrm{P}$ & - & - & 46.9 & - & - & 1.8-Cineol (7.6). & 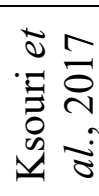 \\
\hline Batna & $\mathrm{P}$ & $\leq 11.2$ & $\leq 10.5$ & $\leq 22.4$ & $\begin{array}{c}\leq \\
19.4\end{array}$ & $\leq 16.8$ & $\begin{array}{c}6- \\
\text { Methoxyelemicine } \\
(\leq 58.2)\end{array}$ & \\
\hline Biskra & $\mathrm{P}$ & $\begin{array}{l}7.2- \\
11.2\end{array}$ & $\begin{array}{l}19.9- \\
28.0\end{array}$ & - & $\begin{array}{c}\leq \\
20.1\end{array}$ & $\leq 18$ & Elemicine $(\leq 29.1)$ & \\
\hline Bechar & $\mathrm{P}$ & - & 32.4 & 19.1 & - & - & $\begin{array}{c}6- \\
\text { Methoxyelemicine } \\
(27.4) .\end{array}$ & \\
\hline Ghardaïa & $\mathrm{P}$ & $\begin{array}{l}2.8- \\
17.1\end{array}$ & $\leq 31.3$ & $\begin{array}{l}9.2- \\
26.7\end{array}$ & $\begin{array}{c}\leq \\
13.6\end{array}$ & - & $\begin{array}{c}6- \\
\text { Methoxyelemicine } \\
(\leq 29.4) ; \alpha- \\
\text { Phellandrene }(\leq \\
15.4) ; \beta- \\
\text { Phellandrene }(\leq \\
7.9) ; \beta \text {-Pinene }(\leq \\
7.1) .\end{array}$ & 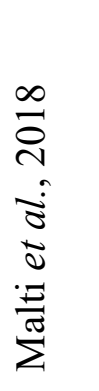 \\
\hline Tunisia & $\mathrm{P}$ & 32.0 & 17.2 & - & - & - & $\begin{array}{c}\Delta \text {-3-Carene (16.9); } \\
\alpha \text {-Thujene (13.7); } \\
\text { Ocimene (9.8). }\end{array}$ & 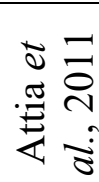 \\
\hline
\end{tabular}

P: Aerial parts; S: Stems; F: Flowers; d: seeds; n.r: not reported.

Previous research on the chemical composition of $P$. Scoparius essential oils obtained by HD and SD from different origins is summarized in (Table 2). It is noted that no study on the essential oil of P. Scoparius as a function of extraction method has been performed to-date. As mentioned above, a significant qualitative and quantitative 
difference in the chemical compositions was observed. As shown in (Table 2), most samples, whether from Tunisia or from different Algerian regions (M'sila, Batna, Beskra and Souk Ahras) were characterized by a high amount $\alpha$-Pinene (8.3-35.8\%) and Sabinene (14.8-34.4 \%) (Lograda et al., 2013; Chikhoune et al., 2016; Attia et al., 2011). Otherwise, essential oils obtained from seeds and stems essential oils from Oum El Bouaghi were dominated by apiol (52.8\% and $15 \%$, respectively) and $\alpha$-pinene (11\% and $34 \%$, respectively). Bornyl acetate was present with large amount only in the seeds oil (21\%) (Vernin et al., 1999). Limonene (32.7$66.5 \%$ ), Myristicin (up to $31.1 \%$ ), dillapiol (up to $23 \%$ ) and $\alpha$-pinene $(4.4-11.2 \%)$ were the main compounds of the aerial part essential oil from Ghardaia (Gourine et al., 2011), while, Germacrene D (12.7\%) was the major compound of the stems oil and Caryophyllene oxide $(12.2 \%)$ the major compound of the seeds from same region (Ghardaïa). These two samples were also characterized by similar amounts of limonene, myristicin and $\alpha$-pinene (6.8-9.8\% and 8.2-11.2\%, respectively for stems and seeds oils) (Vérité and Nacer, 2003). Samples from Djelfa and Laghouat showed similar chemical profiles, dominated by $\alpha$ pinene (23.7-35.8\%), dillapiol (9.9-47.3\%), Limonene (up to $30.0 \%$ ), bornyl acetate (3.09.6\%) and $\beta$-Pinene (up to 5.3\%). However, myristicin was present in high amounts only in the oil obtained from Djelfa (up to 18.2\%) (Gourine et al., 2011). Myristicin $24.1 \%$ and $\alpha-$ pinene $(14.4 \%)$ were also the primary compounds in the flours oil from M'sila, followed by $\alpha$-Phellandrene (15.6\%) (Smaili et al., 2011). The essential oils of P. Scoparius, isolated from Batna, Bechar and Ghardaïa, contained the same dominant compounds: 6Methoxyelemicine $(0.0-59.6 \%)$, sabinene $(0.8-$ $55.6 \%)$, limonene $(0.3-44.0 \%)$, myristicin $(0.0-$ $32.4 \%)$ and $\alpha$-pinene $(0.7-31.0 \%)$. Dillapiol was found as a principal compound only in the oil from Batna (up to 16.8\%) (Malti et al., 2018). 6-methoxyelemicine was identified neither in our work, nor in other reports. In the same study, the samples coming from Biskra were dominated by sabinene (19.9-28.0\%), elemicine (up to $29.1 \%$ ) and myristicin (up to $20.1 \%$ ), while dillapiol and $\alpha$-pinene were also present with appreciable percentages (1.4-18\% and 7.2$11.2 \%$, respectively) (Malti et al., 2018). Limonene $(46.9 \%)$ was the predominant compound in the sample from Tamenrasset, followed by 1.8-Cineol (7.6) (Ksouri et al., 2017). In contrast, $\alpha$-pinene (12.1\%), EpiBicyclosesqui phellandrene $(8.4 \%)$, Eremophilene (8.2\%) and $\gamma$-Cadinene (6.3\%) were the main components of the hydrodistillated oil from the same region (Tamenrasset) (Hammoudi et al., 2015). Furthermore, 7-Methoxy-3-methyl-1-Hisichromen-1-one and methyl propene were identified, along with myristicin, in appreciable amounts only in the oil obtained by SD from Ghardaïa $(10.6 \%, \quad 9.9 \%$ and $12.1 \%$, respectively) (Chikhoune et al., 2016).

\subsection{Acute Toxicity Test}

The oral administration of $P$. Scoparius essential oils in doses of $2000 \mathrm{mg} / \mathrm{kg}$ (limited dose) did not cause any mortality in treated mice for 14 days following the oral administration. According to Hodge and Sterner scale (Hodge, 1943), P. Scoparius essential oil can be classified as non-toxic.

\subsection{Anti-inflammatory activity}

In the present investigation, the antiinflammatory activity of Diclofenac and $P$. Scoparius essential oils extracted by HD, MAE and SD was determined based on the carrageenan induced edema test. The results obtained are expressed in triplicate in (Table 3).

Our results showed that all the studied samples exhibited anti-inflammatory activity in a dose-dependent manner. In fact, the concentration had a significant effect on the extraction methods $(p<0.05)$ with the exception of MAE $(p=0.308)$. The groups treated with essential oils at a dose of $50 \mathrm{mg} / \mathrm{kg}$ showed the lowest inhibition percentages of carrageenaninduced edema $(25.31 \pm 0.85-40.64 \pm 2.36 \%)$ compared to the groups treated with doses of $100 \mathrm{mg} / \mathrm{kg}(27.75 \pm 0.53-44.36 \pm 2.30 \%)$. 
Table 3. Effects of $P$. Scoparius essential oils and Diclofenac on carrageenan-induced hind paw edema in mice

\begin{tabular}{|l|l|l|l|l|}
\hline \multirow{2}{*}{ Doses (mg/kg) } & \multicolumn{4}{|c|}{ Inhibition (\%) } \\
\cline { 2 - 5 } & HD & MAE & SD & Diclofenac \\
\hline 50 & $25.31 \pm 0.85^{\mathrm{c}, \mathrm{C}}$ & $40.64 \pm 2.36^{\mathrm{a}, \mathrm{A}}$ & $31.37 \pm 3.38^{\mathrm{b}, \mathrm{B}}$ & $42.69 \pm 3.04^{\mathrm{a}, \mathrm{C}}$ \\
\hline 100 & $27.75 \pm 0.53^{\mathrm{b}, \mathrm{B}}$ & $44.36 \pm 2.30^{\mathrm{a}, \mathrm{A}}$ & $33.76 \pm 4.33^{\mathrm{b}, \mathrm{B}}$ & $63.32 \pm 1.83^{\mathrm{a}, \mathrm{B}}$ \\
\hline 150 & $55.68 \pm 1.30^{\mathrm{b}, \mathrm{A}}$ & $48.15 \pm 2.82^{\mathrm{c}, \mathrm{A}}$ & $51.09 \pm 3.68^{\mathrm{bc}, \mathrm{A}}$ & $69.01 \pm 1.00^{\mathrm{a}, \mathrm{A}}$ \\
\hline
\end{tabular}

Values are averages \pm standard deviation of triplicate analysis.

Data in the same column having different capital letters are significantly different $(\mathrm{P}<0.05)$ among different concentrations.

Data in the same row having different lower-case letters are significantly different $(\mathrm{P}<0.05)$ among different essential oil extraction method.

Results are ranked in ascending order; $\mathrm{a}>\mathrm{b}>\mathrm{c} ; \mathrm{A}>\mathrm{B}>\mathrm{C}$.

The maximum activity was observed at 150 $\mathrm{mg} / \mathrm{kg}$ for all samples $(48.15 \pm 2.82-55.68 \pm$ $1.30 \%)$. The percentage of inhibition of Diclofenac (50, 100 and $150 \mathrm{mg} / \mathrm{kg})$, taken as a standard drug, also increased from $(42.69 \pm$ $3.04 \%)$ to $(69.01 \pm 1.00 \%)$, with increasing dose. At a dose of $50 \mathrm{mg} / \mathrm{kg}$, HD was less effective than the sample obtained by SD $(25.31$ \pm 0.85 vs $31.37 \pm 3.38 \%)$. However, using MAE, the essential oil showed the highest inhibition percentage $(40.64 \pm 2.36 \%)$. The last value was comparable to that obtained with Diclofenac (42.69 $\pm 3.04 \%)$ and had no significant difference with the standard drug ( $\mathrm{p}$ $=0.743$ ). The same order was observed using samples at a dose of $100 \mathrm{mg} / \mathrm{kg}(27.75 \pm 0.53$, $33.76 \pm 4.33 \%$ and $44.36 \pm 2.30 \%$, respectively for HD, SD and MAE). The results showed that the anti-inflammatory activity of HD vs SD at the dose of $100 \mathrm{mg} / \mathrm{kg}$ has no significant difference $(p=0.667)$. The same is true for MEA and Diclofenac $(\mathrm{p}=0.099)$. In contrast, at a dose of $150 \mathrm{mg} / \mathrm{kg}$, the maximum activity was obtained in HD $(55.68 \pm 1.30 \%)$ and presented no significantly difference from SD ( $\mathrm{p}=0.147)$.

Based on these results, it can be asserted that $P$. Scoparius essential oils have antiinflammatory activity, with significant difference depending on the method of extraction used. This important antiinflammatory activity of $P$. Scoparius essential oils can be attributed to their content of dillapiol, $\beta$-eudesmol, myristicin, Butylidene phtalide $\mathrm{Z}$ and $\alpha$-Pinene. These results validate the use of $P$. Scoparius as an anti-inflammatory in traditional Algerian medicine.

\subsection{Antibacterial assays}

The antimicrobial activity of essential oils was determined against seven bacteria strains and two yeasts using the disk diffusion method. This activity is estimated based on the diameter of inhibition zones. Results are presented in (Table 4) for different extraction methods.

Our data showed that $P$. Scoparius essential oils generally possessed a weak to moderate activity against yeasts (inhibition zone from $7.1 \pm 0.1$ to $13.6 \pm 0.4 \mathrm{~mm})$ as well as grampositive bacteria (inhibition zone from $8.2 \pm 0.1$ to $21.8 \pm 0.3 \mathrm{~mm}$ ).

$S$. lutea was the most affected by $P$. Scoparius essential oils; the oil extracted by HD showed the largest zone of inhibition (inhibition zone $=21.8 \pm 0.3 \mathrm{~mm}$ ) as compared to those obtained by MAE (inhibition zone $=19.8 \pm 0.7$ $\mathrm{mm}$ ) and by SD (inhibition zone $=14.7 \pm 0.6$ $\mathrm{mm}$ ). Similarly, the tested oils exhibited weak activity against $S$. aureus (inhibition zone: $8.2 \pm 0.1-11.0 \pm 0.1 \mathrm{~mm})$ and $B$. subtilus (inhibition zone: $9.1 \pm 0.6-11.2 \pm 0.2 \mathrm{~mm}$ ), while $S$. epidermidis was more affected by the oils obtained by HD and SD (inhibition zone $\approx 12.7$ $\mathrm{mm}$ ). Dillapiol had no effect against $S$. aureus (Ferreira et al., 2016). In another study; 
Dillapiol had no effect against gram-negative bacteria and weak effect against gram-positive bacteria (Eftekhar et al., 2014). This limited effect of $P$. Scoparius essential oils against $S$. aureus could be due to their high content of dillapiol.

These results were previously reported by Ksouri, A. et al. on B. bastilus (inhibition zone
$=8.3 \pm 1.1 \mathrm{~mm}$ ) while $S$. aureus was more resistant to $P$. scoparius essential oil (inhibition zone $=20.0 \pm 3.0 \mathrm{~mm})($ Ksouri et al., 2017). Kiram, A. et al. who worked on P. scoparius from Biskra (Southeast Algeria) reported stronger oil activity against the same bacterial strain, S. aureus [Kiram et al., 2013).

Table 4. Antimicrobial activity of $P$. Scoparius of essential oils used three different methods extractions.

\begin{tabular}{|l|c|c|c|c|c|c|}
\hline \multirow{3}{*}{$\begin{array}{c}\text { Microorga } \\
\text { nisms }\end{array}$} & \multicolumn{6}{|c|}{ Inhibition zone (mm) } \\
\cline { 2 - 7 } & HD & MAE & SD & $\begin{array}{c}\text { Penicillin } \\
\text { G }\end{array}$ & Oxalin & Amoxypen \\
\cline { 2 - 7 } & \multicolumn{7}{|c|}{} \\
\hline Gram- positive bacteria \\
\hline S. aureus & $8.2 \pm 0.1^{\mathrm{b}, \mathrm{F}}$ & $8.2 \pm 0.1^{\mathrm{b}, \mathrm{D}}$ & $11.1 \pm 0.1^{\mathrm{a}, \mathrm{B}}$ & 40 & 40.6 & 38 \\
\hline S. epidermidis & $12.4 \pm 0.3^{\mathrm{a}, \mathrm{C}}$ & $10.4 \pm 0.2^{\mathrm{a}, \mathrm{C}}$ & $12.1 \pm 0.6^{\mathrm{a}, \mathrm{AB}}$ & 14.6 & 21.8 & 17.6 \\
\hline S. lutea & $21.8 \pm 0.3^{\mathrm{a}, \mathrm{A}}$ & $19.8 \pm 0.7^{\mathrm{a}, \mathrm{A}}$ & $14.7 \pm 0.6^{\mathrm{b}, \mathrm{A}}$ & $>45$ & $>45$ & $>45$ \\
\hline B. subtilus & $11.2 \pm 0.2^{\mathrm{a}, \mathrm{D}}$ & $9.1 \pm 0.6^{\mathrm{b}, \mathrm{D}}$ & $9.5 \pm 0.1^{\mathrm{b}, \mathrm{C}}$ & 35 & 40 & 34 \\
\hline Gram-negative bacteria \\
\hline E. faecalis & - & - & - & 12 & 25 & 26 \\
\hline E. coli & - & - & - & 31 & 16.9 & 40 \\
\hline P. aeruginosa & - & - & - & - & 17 & 19 \\
\hline Yeast & - & - & - & - & - \\
\hline C. albicans & $11.5 \pm 0.3^{\mathrm{b}, \mathrm{D}}$ & $11.3 \pm 0.2^{\mathrm{b}, \mathrm{B}}$ & $13.4 \pm 0.2^{\mathrm{a}, \mathrm{A}}$ & - & - & - \\
\hline S. cerevisiae & $13.6 \pm 0.4^{\mathrm{a}, \mathrm{C}}$ & $7.1 \pm 0.1^{\mathrm{b}, \mathrm{E}}$ & $12.0 \pm 0.1^{\mathrm{a}, \mathrm{A}}$ & - & - & \\
\hline
\end{tabular}

Values are averages \pm standard deviation of triplicate analysis, zone of inhibition in $\mathrm{mm} \pm$ standard deviation beyond well diameter $(6 \mathrm{~mm})$, data in the same column having different capital letters are significantly different $(\mathrm{P}<0.05)$ among different microorganisms, data in the same row having different lower case letters are significantly different $(\mathrm{P}<0.05)$ among different essential oil extraction method, results are ranked in ascending order; $\mathrm{a}>\mathrm{b} ; \mathrm{A}>\mathrm{B}>\mathrm{C}>\mathrm{D}>\mathrm{E}>\mathrm{F}$, -: not action

Alternatively, P. scoparius essential oils showed no activity (inhibition zone $=0 \mathrm{~mm}$ ) against gram-negative bacteria $E$. faecalis, $E$. coli and $P$. aeruginosa. Unlike gram-positive bacteria, it is known that gram-negative bacteria have a very high resistance to essential oils due to their wall made up of a second external barrier (Okoh et al., 2010; Dhouioui et al., 2016). The same results were obtained by other authors who reported that $P$. Scoparius essential oils generally showed limited activity against $P$. aeruginosa, E coli and E. faecalis (Ksouri et al., 2017; Ferreira et al., 2017).

Our findings displayed a strong significant difference against $S$. aureus and $S$. cerevisiae between MEA and SD $(\mathrm{p}<0.001)$, and no significant difference between HD and MEA against strains ( $>00.05)$, except for $B$. subtilus and $S$. cerevisiae. The comparison of HD vs SD showed that the extraction method influenced the antimicrobial activity except for $S$. epidermidis and $S$. cerevisiae $(\mathrm{p}<0.05)$. This could be due to the high content of hydrocarbon monoterpene since the antimicrobial activity of the essential oil could be linked to the presence of a-pinene (Bourkhiss et al., 2007; Abi-ayada et al., 2011; Amarti et al., 2011)); although minor molecules can also produce antibacterial activity, contributing to the activity of essential oils (Okoh et al., 2010). 


\section{Conclusions}

The effects of extraction methods on $P$. Scoparius essential oil compositions and their anti-inflammatory and antimicrobial activities are investigated here for the first time. The chemical compositions of the essential oils obtained are qualitatively similar, but with significant differences in the abundance of major compounds. P. Scoparius essential oils are non-toxic and exhibit important antiinflammatory activity and weak antibacterial activity. The extraction method significantly affected the anti-inflammatory and antibacterial activities. It is very important to continue this research and find a correlation between the extraction techniques and their effects on the chemical composition and biological activities.

\section{References}

Abdellah, H., Sahki, R. E. (2004). Eds. Le Hoggar: Promenade botanique, Herbaceous species.

Abi-Ayada, F. Z., Abi Ayad, M., Lazzouni, H. A., Rebiahi, S., Bessiere. A. (2011). Antibacterial Activity of Essential oil extracted from leaves of Tetraclinis articulata (Vahl) Master from Algeria flora, Journal of Microbiology and Biotechnology Research, 1, 1-6.

Adams, R.P. (2007). Identification of Essential Oil Components by Gas Chromatography/Mass Spectrometry. ( $4^{\text {th }}$ ed.). Allured Pub Corp.

Amarti, F., Satrani, B., Ghanmi, M. Farah, A., Aafi, A., Aarab, L., El Ajjouri, M., Chaouch, A. (2010). Composition chimique et activité antimicrobienne des huiles essentielles de Thymus algeriensis Boiss. \& Reut. et Thymus ciliatus (Desf.) Benth. du Maroc. Biotechnology, Agronomy, Society and Environment / Biotechnology, Agronomy, Society and Environment, 14(1), 141-148.

Attia, S., Grissa, K.L., Lognay, G., Heuskin, S., Mailleux, A.C., Hance, T. (2011). Chemical composition and acaricidal properties of Deverra Scoparia essential oil (Araliales: Apiaceae) and blends of its major constituents against Tetranychus urticae
(Acari: Tetranychidae). Journal of Economic entomology, 104(4), 1220-1228.

Belkacemi, D., Kalla, A., Rouabhi R., Amara, D. (2016). Effect of Pituranthos Scoparius essential oils on reducing metganogenesis in cheep: in vitro Study. Environmental Science and Indian Journal, 12(3), 118-126.

Benchelah, A. C., Bouziane, H., Maka, M., Ouahes, C. (2000). Fleurs du Sahara : Voyage ethnobotanique avec les Touaregs du Tassili. 2nd eds. Ibis Press, 105-106.

Beoletto, V. G., De las Mercedes Oliva, M., Marioli, J. M., Carezzano, M. E., Demo, M. S. (2016). Antimicrobial Natural Products Against Bacterial Biofilms. Mechanisms and New Antimicrobial Approaches, Antibiotic resistance, Chapitre 14, 291-307.

Boukef, M. K. (1986). Plants in traditional Tunisian medicine. Cultural and technical cooperation agency, 1986, 228-230.

Bourkhiss, M., Ouhssine, M., Hnach, M., Satrani, B., Farah, A. (2007). Composition chimique et bioactivité de l'huile essentielle des rameaux de Tetraclinis articulata. Bulletin of the Bordeaux Pharmacy Society, 146, 75-84.

Chikhoune, A., Pavleca, J. D., Shashkov, M., Berroua, Z., Chebbi, K., Bougherra, H., Zeroual, B., Aliane, K., Gagaoua, M., Boudjellal, A., Vovk, I., Križman, M. (2016). Antioxidant effect induced by the essential oil of Pituranthos Scoparius in a formulation of a whey spread emulsion. Journal of Food Processing and Preservation, 41(5), 1-12

Council of Europe, Pharmacopée Européenne, (2010). $7^{\text {th }}$ Eds. Directorate for the Quality of Medicines \& HealthCare of the Council of Europe, Strasbourg, France.

Dhouioui, M., Boulila, A., Chaabane, H., Saïd Zina, M., Casabianca, H. (2016). Seasonal changes in essential oil composition of Aristolochia longa L. ssppaucinervis Batt. (Aristolochiaceae) roots and its antimicrobial activity. Industrial Crops and Products, 83, 301-306.

Eftekhar, F., Yousefzadi, M., Azizian, D., Sonboli,A., Salehi, P. (2005). Essential Oil 
Composition and Antimicrobial Activity of Diplotaenia damavandica. Zeitschrift für Naturforschung C, 60(11-12), 821-825.

Elyemni, M., Louaste, B., Nechad, I., Elkamli, T., Bouia, A., Taleb, M., Chaouch, M., Eloutassi, N. (2019). Extraction of Essential Oils of Rosmarinus officinalis L. by Two Different Methods: Hydrodistillation and Microwave Assisted Hydrodistillation. The Scientific World Journal, 1-6.

Ferreira, R. G., C. Monteiro M. C., da Silva, J. K. R., Maia J. G. S. (2016). Antifungal Action of the Dillapiole-rich Oil of Piper aduncum against Dermatomycoses Caused by Filamentous Fungi. Journal of Advances in Medicine and Medical Research, 15(12), $1-10$.

Gourine, N., Merrad, B., Yousfi, M., Stocker, P., Gaydou, E.M. (2011). Chemical composition of the essential oil of Pituranthos Scoparius. Natural Product Communications, 6(8), 1151-1154.

Hammiche, V., Maiza K. (2006). Traditional medicine in Central Sahara: pharmacopoeia of Tassili N'ajjer. Journal of Ethnopharmacology, 105(3), 358-367.

Hammoudi, R., Dehak, K., Hadj Mahammed, M., Didi Ouldelhadj, M. (2015) Composition chimique et activité antioxydante des huiles essentielles de Deverra Scoparia Coss. \& Dur. (Apiaceae). Lebanese Science Journal, 16(2), 27-36.

Hodge, H.C., Sterner, J.H. (1943). Determination of substance acute toxicity by LD50. American Industrial Hygiene Association Journal, 9, 93-96.

Karakaya, S., Nehir El, S., Karagozlu, N., Sahin, S., Sumnu, G., Bayramoglu, B. (2014). Microwave-assisted hydrodistillation of essential oil from rosemary. Journal of Food Science and Technology, 51(6), 1056-1065.

Kiram, A., Ramdani, M., Zeraid, A. (2013). Etude Phytochimique et de L'activité Antimicrobienne des Huiles Essentielles de Pituranthos Scoparius de la Region de Biskra (Sud-Est Algérien). Tunisian Journal of Medecinal Plants and Natural Products, 10, 1-6.
Ksouri, A., Dob, T., Belkebir, A., Dahmane, D., Nouasri, A. (2017). Volatile compounds and biological activities of aerial parts of Pituranthos Scoparius (Coss and Dur) Schinz (Apiaceae) from Hoggar, southern Algeria. Tropical Journal of Pharmaceutical Research, 16(1), 51-58.

Levy, L. (1969). Carrageenan paw edema in the mouse, Life Sciences, 8(1), 601-606.

Lograda, T., Ramdani, M., Kiram, A., Chalard, P., Figueredo, G. (2013). Variation of essential oils composition of Pituranthos Scoparius in Algeria. Global journal of research on medicinal plants and indigenous medicine, 2(1), 1-9.

Lo Presti, M., Ragusa, S., Trozzi, A., Dugo, P., Visinoni, F., Fazio, A., Dugo, G., Mondello, L. (2005). A comparison between different techniques for the isolation of rosemary essential oil. Journal of Separation Science. 28(3), 273-280.

Lorke, D.A. (1983). New approach to practical acute toxicity, Archives of Toxicology, (pp. $53)$.

Lucchesi, M. E., Chemat, F., Smadja, J. (2004). Solvent-free microwave extraction of essential oil from aromatic herbs: comparison with conventional hydrodistillation. Journal of Chromatography, 1043(2), 323-327.

Malti, C., Boussaïd, M., Belyagoubi, L., Paoli, M., Gibernau, M., Tomi, F., AtikBekkara, F., Bekhechi, C. (2018). Chemical Variability of the Essential Oil $\mathrm{O} f$ Pituranthos Scoparius from Algeria, Chemistry \& Biodiversity, 15(7).

Mohammedi, H., Mecherara-Idjeri, S., FoudilCherif, Y., Hassani, H. (2015). Chemical composition and antioxidant activity of essential oils from Algerian daucus carota L. subsp. carota aerial parts. Journal of Essential Oil-Bearing Plants, 18 (4), 873883.

Mohammedi, H., Mecherara-Idjeri, S., Hassani, H. (2016). Variability in essential oil composition, antioxidant and antimicrobial activities of Ruta montana L. collected from different geographical regions in Algeria. 
Journal of essential oil research, 32 (1), 88101.

Morad, S., Fazlali, A., Hamedi. A. (2018). Microwave-assisted hydro-distillation of essential oil from rosemary: Comparison with traditional distillation. Avicenna Journal of Medical Biotechnology, 10(1), 22-28.

National Committee for Clinical Laboratory Standards. (1997). Performance standards for antimicrobial disk susceptibility tests. Approved standard M2-A6. Wayne, Pa: National Committee for Clinical Laboratory Standards.

Okoh, O.O., Sadimenko, A. P., Afolayan, A. J. (2010). Comparative evaluation of the antibacterial activities of the essential oils of Rosmarinus officinalis L. obtained by hydrodistillation and solvent free microwave extraction methods. Food Chemistry, 120 (1), 308-312.

Ould el hadj, M., Hadj mahammed, M., Zabeiro, H. (2003). Place des plantes spontanées dans la médicine traditionnelle de la région de Ouargla (Sahara septentrional est). Courrier du Savoir, 47-51.

Ozenda, P. (2004). Flore et végétation du Sahara. ( (rd $^{\text {rd }}$ ed.). CNRS, 663.

Pushpangadan, P., George, V. (2012). Handbook of Herbs and Spices. $2^{\text {nd }}$ Eds, Vol. 1, Woodhead Publishing Series in Food Science, Technology and Nutrition, 55-72.

Quezel, P., Santa, S. (1962). Nouvelle flore de l'Algérie et des régions désertiques. (CNRS ed.). (pp. 1170).

Smaili, T., Zellagui, A. Gherraf, N., Flamini, G., Cioni, P. L. (2011). Essential oil content of the flowers of Pituranthos Scoparius in Algeria Medicinal plant, International Journal of Phytomedicines and Related Industries, 3(2), 177-179.

Vernin, G., Ghiglione, C., Mostafa, D., Parkanyi, C. (1999). GC/MS Analysis of volatile constituents of essential oils of
Pituranthos Scoparius, Journal of essential oil research, 11, 673-676.

Vérité, P., Nacer, A. (2003). Composition of seeds and stems and essential oils of Pituranthos Scoparius (Coss. \& Dur.) Schinz. Flavour and Fragrance Journal, 19, 562-564.

Wang, Z., Ding, L., Li, T., Zhou, X., Wang, L., Zhang, H.Q. (2006). Improved solvent-free microwave extraction of essential oil from dried Cuminum cyminum L. and Zanthoxylum bungeanum Maxim. Journal of Chromatography A, 1102(1-2), 11-17.

\section{Acknowledgements}

We would like to especially thank the Department of Pharmacotoxicology-Saidal of Medea, Algeria, for laboratory materials support and for hosting the pharmacological activities in their laboratories. 